\title{
Ct Cardiac Imaging for Evaluation and Treatment of Ischemic Heart Disease
}

\author{
Faruk Erzengin ${ }^{1}$,, Evren Bursuk ${ }^{2}$ \\ ${ }^{1}$ Department of Cardiology, Previous Dean, University of Istanbul, Istanbul Medical Faculty, Istanbul, Turkey \\ ${ }^{2}$ Program of Biomedical Technologies, University of Istanbul, Vocational School of Technical Sciences, Istanbul, Turkey
}

Email address:

farukerzengin@gmail.com (F. Erzengin), farukerzengin@istanbul.edu.tr (F. Erzengin), bursukev@istanbul.edu.tr (E. Bursuk), phoenix2007x@gmail.com (E. Bursuk)

\section{To cite this article:}

Faruk Erzengin, Evren Bursuk. Ct Cardiac Imaging for Evaluation and Treatment of Ischemic Heart Disease. American Journal of Internal Medicine. Vol. 3, No. 4, 2015, pp. 165-179. doi: 10.11648/j.ajim.20150304.14

\begin{abstract}
The formation of atherosclerotic and calcified plaques begins and develops not only below the endothelium (intimae), but also in the adventitia. Multislice Computed Tomography (MSCT= Cardiac CT) is a very important tool for the diagnosis and treatment of the atherosclerotic calcification in the coronary arteries. Due to rapid improvement in technology, MSCT techniques have also progressed quickly, leading to the development of the 640 slice MSCT. Following the development of this advanced non-invasive technology (640 slice), came up from stage 4C than to the $1 \mathrm{~A}$ level. Compared to older technologies (e.g. 64, 128 and 320 slice MSCT), the 640 slice MSCT provides various advantages such as higher lateral resolution, lower radiation, and the ability to obtain results more rapidly and accurately. We also introduce a special four cases diagnosed by MSCT, two of them silent ischemia, one of them acute asymptomatic myocardial infarction, and the last one angina pectoris with myocardial bridge(respectively, a 88 year old man, 62 year old man, 66 year old man and 53 year old woman). It was observed that a new innovative combined drug (polypill) is able to prevent the formation of atherosclerotic and calcified plaque of arteries, and to regress pre-existing ones.
\end{abstract}

Keywords: Adventitial atherosclerotic calcifications, Formation of Atherosclerosis on the Adventitia, MSCT, Innovative Treatment for Atherosclerosis, Erzengin's Polypill

\section{Introduction}

Owing to the increasing body of knowledge regarding atherosclerosis, it is nowadays accepted that atherosclerosis develops not only beneath the intimae (subendothelium) or media, but also in the adventitial epithelium of the coronary arteries. In addition, this adventitial atherosclerosis progresses more severely than others. Dr. Erzengin determined that MSCT is an important tool for diagnosis and treatment of adventitial atherosclerosis, especially for early diagnosis of silent myocardial ischemia and asymptomatic myocardial infarction. In parallel to rapid improvements and advances in technology, MSCT techniques have also progressed rapidly, leading to the development of the 640 slice MSCT. Following the development of this advanced non-invasive technology, came up from stage $4 \mathrm{C}$ than to the $1 \mathrm{~A}$ level. Compared to older technologies (e.g. 64, 128 and 320 slice MSCT), the 640 slice MSCT provides various advantages such as higher lateral resolution, lower radiation, and the ability to obtain results more rapidly and accurately.

Despite the steady progress in the treatment of atherosclerotic and atherothrombotic cardiovascular diseases, coronary artery diseases and myocardial infarctions continue to be significant causes of morbidity and mortality, especially in industrialized countries. The term atherosclerosis refers to the thickened and hardened lesions, which have lipids and calcifications in the intimae and media of elastic and muscular arteries. To date, the primary cause of arterial atherosclerotic calcification has not yet been elucidated. It is generally accepted that atherosclerotic and calcified lesions first appear and develop within the innermost layer of the arteries (at the intimae). In 1995, the American Heart Association (AHA) described that the earliest lesions of atherosclerosis (fatty streaks or type III lesions) are present in the intimae of the aorta from childhood. Today, it is known that atherosclerosis begins as early as fetal life, especially in the fetuses of hypercholesterolemic mothers [1]. Formation and 
progression of atherosclerotic plaques and calcifications in all arterial beds of intimae have been well documented by many authors, such as Fuster and Falk [1, 2, 3, 4], who subdivided the formation and progression of these plaques into several phases summarized below. Most lipids deposited in atherosclerotic lesions (atherosclerotic components) are derived from plasma low-density lipoproteins (LDLs) that enter the vessel wall through injured or dysfunctional endothelium. Within the normal population, fatty streaks often appear in the aorta and coronary arteries starting from the ages of 5 to 10 ; these are considered as the initial/starting points of plaque development. Type I lesions consist of macrophage-derived foam cells that contain lipid droplets; Type II lesions contain macrophages and smooth muscle cells with extracellular lipid deposits; Type III lesions contain smooth muscle cells surrounded by extracellular connective tissue, fibrils, and lipid deposits; Type IV plaques consist of confluent cellular lesions with large amounts of extracellular lipid intermixed with fibrous tissue; and Type Va plaques possess an extracellular lipid core covered by a thin fibrous cap. Both phase III and IV plaques can evolve into phase $\mathrm{V}$ fibrotic plaques, characterized by type $\mathrm{Vb}$ or Vc lesions, with or without predominant calcification. Type VI lesions are occlusive thrombi overlying a superficial erosion of markedly stenotic and fibrocalcific phase $\mathrm{V}$ plaques $[5,6,7]$. In Phases II to $\mathrm{V}$, vulnerable lipid-rich plaques are prone to disruption due to their high lipid content. Raised plaques appear later, generally by 20 years of age, and are present in areas such as the endothelium of proximal left anterior descending (LAD) coronary artery, where fatty streaks are most prevalent in early life $[3,8]$. When fully opened, the total surface of all arteries in humans is approximately 800 square meters. Arteries produce more than 250 active substances. There are several types of plaque: Some are flat yellow dots or lines (fatty streaks), while others are raised above the surface as oval humps, which range in color from white to yellow (raised fibro lipid plaques) $[5,6]$. With early atherosclerotic lesions (fatty streaks or type III lesions) in the aortic root and coronary arteries, the lesion consists of lipid-laden monocyte-derived macrophage foam cells and a few $\mathrm{T}$ lymphocytes beneath an intact endothelium. It is generally accepted that only endothelial cells, monocyte-derived macrophages or foam cells, and a few $\mathrm{T}$ cells participate in the early inflammatory and immune responses that give rise to the early atherosclerotic lesions. These early atherosclerotic lesions (fatty streaks or type III lesions) represent a dynamic balance of the entry and exit of lipoproteins by endothelium injury, resulting in a predominance of lipoprotein exit and final scarring. During the progression of the disease, the oxidative modified LDL (oxLDL), the inflammatory and immune response is accompanied by a fibro-proliferative response in which the vascular smooth muscle cells play dominant roles $[9,10,11$, 12]. OxLDL has many proinflammatory properties, which explain the local up regulation of inducible endothelial cell adhesion molecules - even before lesion formation - in hypercholesterolemic atherosclerosis and atherothrombotic events. OxLDL has proinflammatory and cytotoxic effects. It is recognized by the macrophage scavenger receptor, promoting intracellular lipid accumulation and foam cell formation. Such as the endothelial dysfunction or/activation (vascular cell adhesion molecule 1), monocyte adherence, injuring lipoprotein retention, oxLDL, inflammatory/immune response, macrophage scavenger foam cell formation, endothelial shear stress, turbulent flow, high blood pressure on the vessels' walls and the role of nitric oxide (NO), prostacyclin, endothelin-1, acetylcholine, vascular cell adhesion molecule-1 (CAM-1), cell adhesive molecules (CAM or surface glycoproteins), intercellular adhesion molecule-1 (ICAM-1) promote or enter though the injured or dysfunctional endothelium and subendothelial lipid accumulation and at the end lipid core formations occur. Monocytes (macrophages) adhere to the surface of endothelium (intimae) through specific molecules, such as the monocyte chemotactic protein-1(MCP-1) and macrophage colony-stimulating factor (M-CSF) [1]. Before or after their death, macrophage or foam cells can release various substances, including oxLDL and free radicals. During chronic minimal endothelial injury or dysfunction, plasma low-density proteins enter through the injured endothelium, leading to accumulation of lipids (oxLDL), while the macrophages produce atheroma masses or atheromatous component. The extent to which atheroma masses or atheromatous components are produced depends on various factors such as genetics, diabetes, hypercholesterolemia, tobacco smoking, hypertension, abdominal obesity, gender, aging, high plasma uric acid level, chemical irritants, infections, high level svCRP and fibrinogen, circulating vasoactive amines, and immune complexes. These more than two hundred fifty factors can reach all tunicas of the arteries, as well as the adventitia, through the vasa vasorum and the neighboring epicardial lipid mass.

As mentioned in an international report on the social aspects of atherosclerosis, the risks factors for atherosclerotic vascular diseases that can be classified as major risk factors and emerging risk factors. Age, sex, high plasma uric acid levels, smoking, hypertension, low level HDL-cholesterol and diabetes are the major risk factors that accelerate the atheromatous process, while emerging risk factors include chemical irritants, infections, high svCRP and fibrinogen levels, circulating vasoactive amines, and immune complexes that are associated with atherosclerotic complications. Thus, patients who have the emerging factors undergo invasive procedures that either involve stent implantation or coronary bypass surgery. Besides, there are also underlying risk factors which lead to the development of the major and emerging risk factors. These underlying risk factors are atherogenic diets, abdominal obesity, physical inactivity, and genetic effects [7].

So this classical pathway or famous well known cascade of atherosclerosis brings about the formation of the 
atheroma in 1995 by AHA of atherosclerotic lesions [1, 2, 3, 7]. This pathway of formation of atherosclerosis and calcifications almost universally were accepted in the intimae (under the endothelium). In spite of the fact that, there is no any doubt of this classical pathway or rule of the cascade of formation of atherosclerotic calcifications. Atherosclerosis is a focal disease in the intimae of large and medium sized systemic arteries. It has been well documented that, focal calcification in atherosclerotic plaques is very common in humans' arteries and nicely shown that coronary calcification in adults is almost always atherosclerosis related intimae until today [9]. We have recently shown this with the MSCT method (previously 320 slice; currently 640 slice) using within a device which has a computerized magnifying glass. On the other hand making a comparison between MSCT, coronary angiography, perioperative findings and histopathological data which detected by light and electron microscopy and environmental SEM (By using a microtome, slice samples can be taken, across the walls of samples classical histopathological routine investigation and addition to chemical analysis can be taken by means of energy dispersive X-ray mobile monitoring the surface of specimen by SEM). We strongly emphasized that, the classical cascade of the atherosclerotic and calcified plaques formation appear and develop not only beneath the intimae (subendothelium) or media, but also in adventitial epithelium on the coronary arteries. We have surprisingly determined that the adventitial side of the calcification develops much faster and more severely than that of the intimae and medial side on the arterial vessel walls.

MSCT of coronary angiography with 64 slice technology was first described by Leschka S, et al [8]. Currently, we have performed our studies with MSCT at the beginning 64 slice and later on 320 slice with magnifying glass, which are very important tool for the non-invasive evaluation of coronary arterial pathologies. Moreover, the MSCT is an excellent and important technique for showing the location and size of plaque formation and calcification in the arteries $[8,9]$. It has been well documented in the last three decades that the cascade of atherosclerotic plaque formation begins from beneath the endothelial cells of the coronary arteries $[13,14,15]$. The media calcification of the coronary arteries was also described as Mönckeberg's Sclerosis many years ago, and while rare in coronary arteries, it is frequent in other types of arteries (particularly in the arteries of the leg and aorta) $[16,17,18]$. All calcification of arteries are directly related with atherosclerotic burden or component. Until today it has not written yet anything about our findings of adventitial atherosclerosis and calcifications [22,
23]. In a two-year randomized study in 1997 on identical twin yeanlings, we determined that there were significant parallels between umbilical obesity and epicardial lipid mass. Moreover, we demonstrated that the epicardial lipid mass is statistically significantly associated with adventitial localization over the arteries [9, 19].

We have recently shown with MSCT-320 that the formation of atherosclerotic and calcified plaques begin not only in the intimae, but, surprisingly, also under the adventitial epithelium of the coronary arteries. The adventitial calcifications develop more rapidly than the subendothelial (the intimae) calcified plaques. On the other hand, the medial calcifications develop far more frequently on the subendothelium of the aorta and its main branches, such as the carotid, vertebral, cerebral, renal, mesenteric, ilio-femoral, and peripheral arteries. Interestingly, the formation of calcified plaque begins more frequently beneath the subepithelium of the adventitia, and quickly develops towards to the lumen of arteries, following the same classical and accepted cascade on the coronary arteries. These calcifications mostly begin in the middle of the atheromatous component. Cholesterol (particularly oxLDL) and macrophage cells easily arrive to the adventitia vasa vasorums of the coronary arteries from blood and/or by diffusion from epicardial fat accumulation around the heart appears in three different types: a) İntracellular in the heart; b) Epicardial; and c) Pericardial adipose tissue around the heart. Intracellular fat is the microscopic lipid accumulation within the cytoplasm cardiac myocytes, and can result from myocardial ischemia, cell damage and/or cell death. As shown in Figure 1, the epicardial fat tissue is located between the outer wall of the myocardium and the visceral layer of pericardium, while the pericardial fat exists anterior to the epicardial fat layer and therefore located between visceral and parietal pericardium. Due to the close anatomic relation between myocardium and the epicardial fat, the two tissues share the same microcirculation. Through the vasa vasorums, diffusion way or potential interactions through paracrine and vasocrine mechanism between epicardial fat and adventitia of coronary arteries or myocardium are strongly suggested [24,25].

Furthermore, we also demonstrated that previously performed Multi Slice Computed Tomography has played an important role for patients as a guide for detecting size of devices and to perform elective procedure of coronary lesions during implantation of stent in the cat-lab.

Objective: The aim of this study is to cure the atherosclerosis by classical and current medical treatments and also emphasize the importance of MSCT and introduce four special cases. 


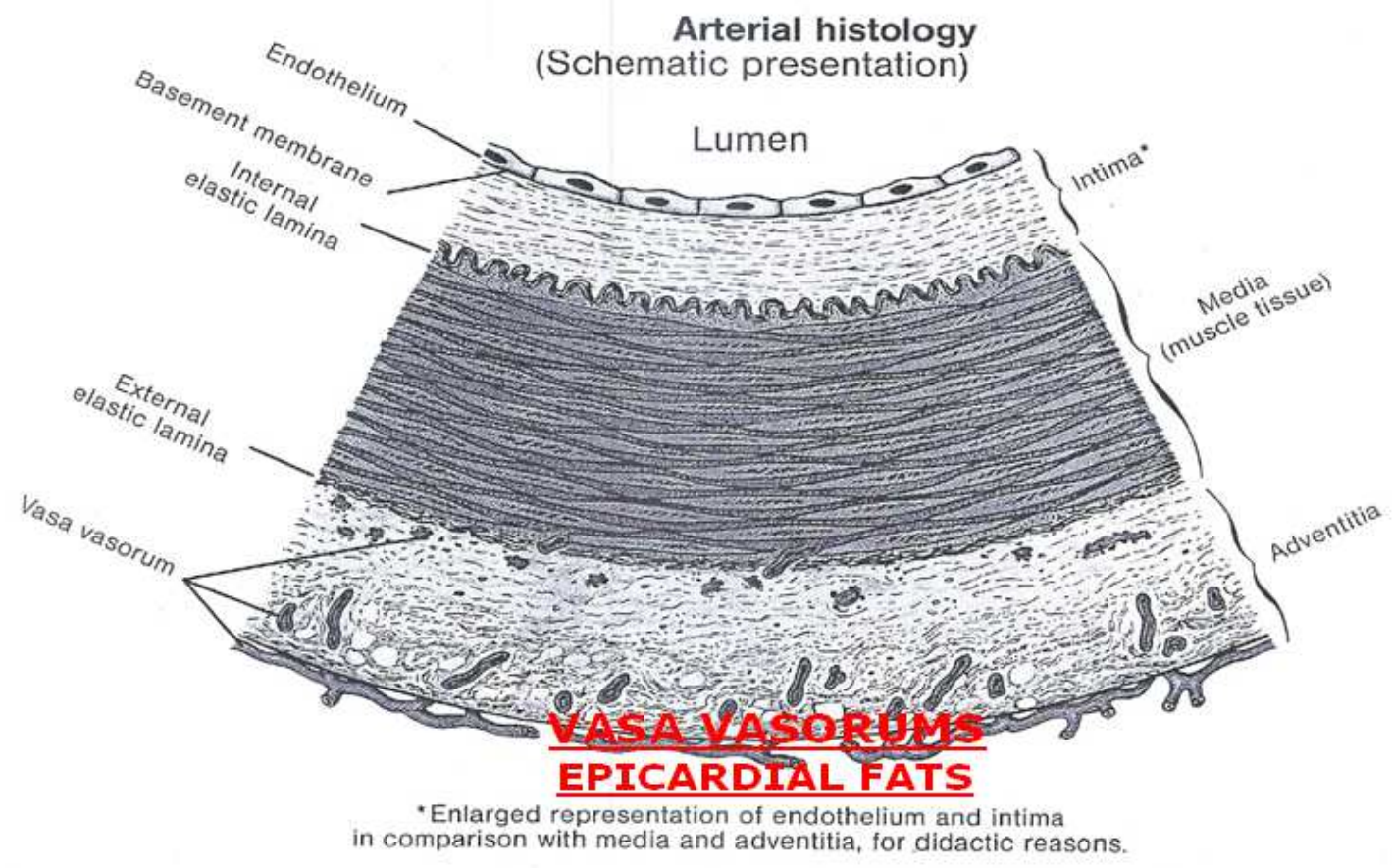

Figure 1. This figure shows epicardial fats tissue, vasa vasorums and tunicas of an artery (endothelium, intimae, tunica elastica interna, media, tunica elastica externa and adventitia).

\section{Material and Methods}

Following animal studies, we examined coronary atherosclerotic calcifications and modern current treatment in 4055 patients at Istanbul Medical Faculty, and focused in particular on adventitial localization since 07.07.1997. Most risk factors apply to all atherosclerotic vascular diseases. In this context, seven different consecutive cases (Fig.4-10) were investigated by MSCT320 and conventional coronary angiography. The atherosclerotic plaques and calcifications appeared and developed beneath the adventitial epithelium through the lumen of vessels on the coronary arteries. Our recent studies showed that in most patients (approximately $51 \%$ ), the calcified plaques started just beneath of the epithelium on the adventitia and progressed towards to the lumen of the arteries. Of course, they can make a severe stenosis or occlusion of arterial lumen at the end. In comparison with IVUS, Stephan Achenbach et al. found a sensitivity of $82 \%$ to detect coronary artery segments containing atherosclerotic plaque in patients without significant coronary artery stenosis [16]. MSCT might be useful for the characterization of human coronary plaque morphology by non-invasively determining tissue density within the lesion. In addition, MSCT is a unique noninvasive method for the detection of coronary atherosclerotic plaque morphology, and for the diagnosis of silent ischemia, lumen narrowing calcification of adventitia [19]. Molecular imaging will most probably assist with the sorting and identification of this complex morphology in the near feature.

We first observed the opposite of what is normally described regarding the atherosclerotic location and pathway, which is that the formation of atherosclerotic plaques begin not only from intimae, but also from adventitial epithelium of the coronary arterial walls. The MSCT- 320 is a unique technique that showed the unknown opposite side of the classical cascade and pathway of atherosclerosis. This finding was described in our randomized comparative coronary studies comparing invasive and non-invasive techniques, which is not described in the literature. We have emphasized that MSCT-320with magnifying glass is an important and unique device, as well as an important tool for non-invasive evaluation of coronary arterial pathologies and plaque formations.

In the first patient, a normal coronary artery taken by MSCT was shown in Fig2. On Figure 3, the adventitial atherosclerotic calcifications (atheromatous components) on the femoral arteries of Guinea pigs have been shown. In the third case (Fig. 4), a soft plaque of atheroma on the right coronary artery of adventitia takes place. In the Figure 5, the atherosclerotic soft and vulnerable plaque (atheromatous component) starting from adventitia of the mid segmental part of the Left Anterior Descending (LAD) artery and multiple small adventitial calcifications were shown that just beneath the subepithelium of the adventitia on the (LAD). In Fig 4-10 have been shown from minimal to severe calcifications and some degree of atheromatous components were shown which started and were taken placed just beneath the epithelium of adventitia and grown towards to the coronary arterial lumen making some degree of obstructions on the Left Main, LAD and Circumflex $(\mathrm{Cx})$ of coronary. MSCT-320 is very useful device for the characterization of human coronary plaque morphology by determining tissue 
density within the lesion non-invasively. Also MSCT is a unique non-invasive method for the diagnosis of silent ischemia and asymptomatic myocardial infarctions due to lumen narrowing calcification of adventitia.

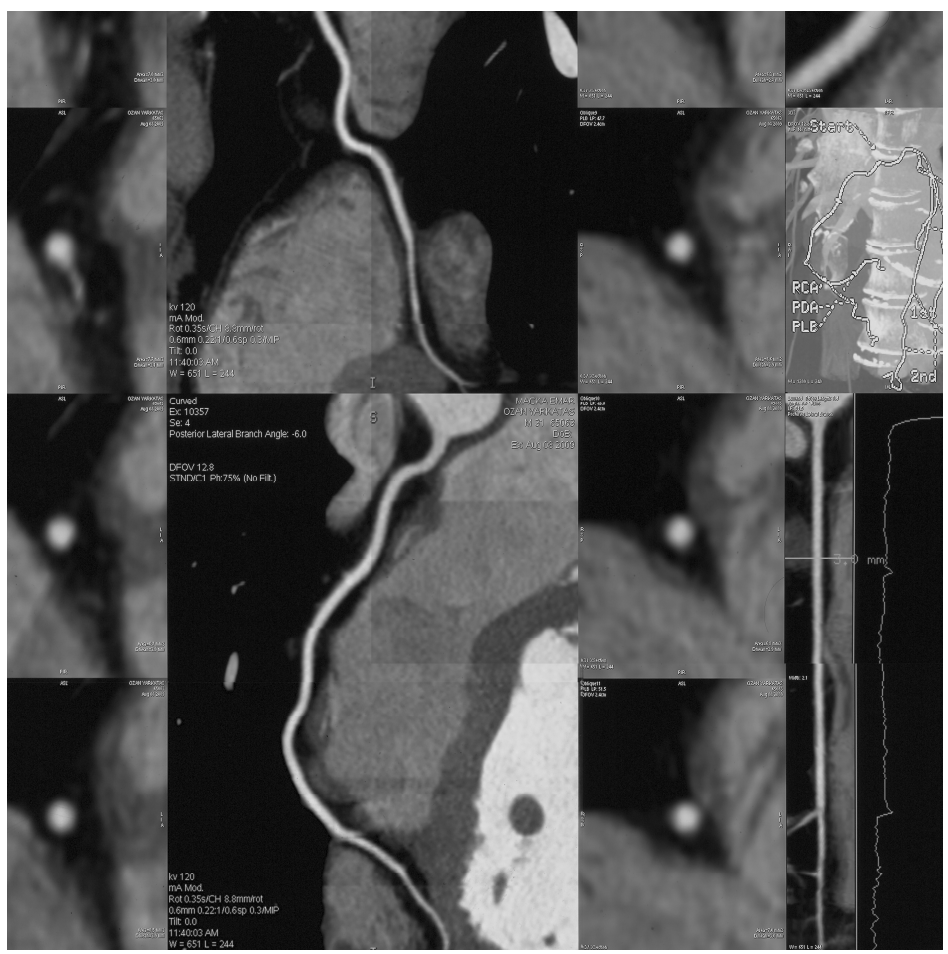

Figure 2. A normal Right Coronary Artery (white arrow) is shown by MSCT with no atherosclerotic plaque or calcification.

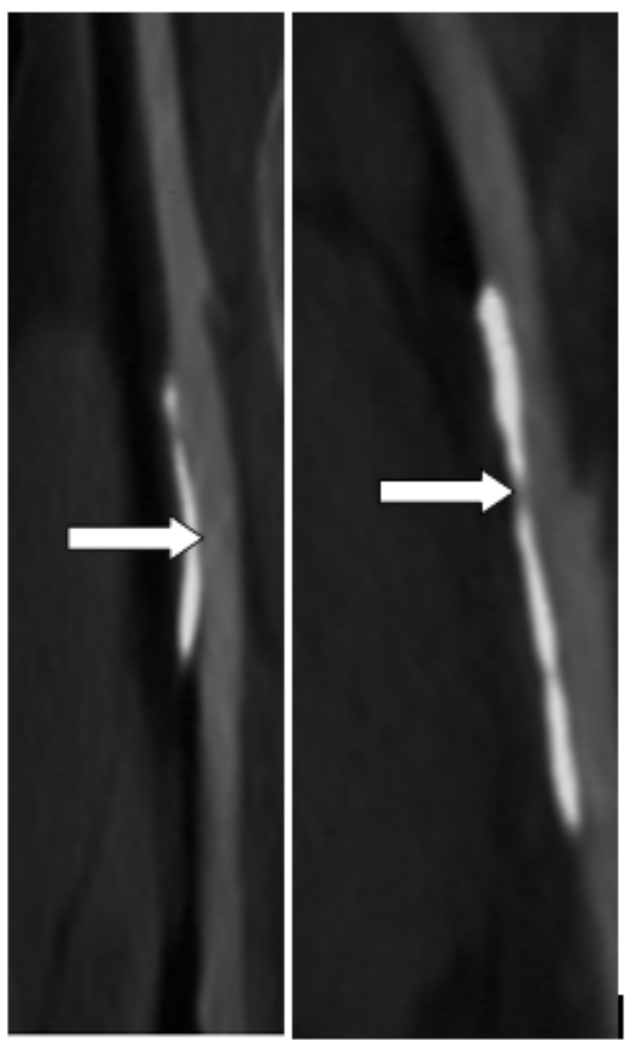

Figure 3. Adventitialatherosclerocalcifications (atheromatous components) of on the femoral arteries of Guinea pigs The grey areas are soft plaque (lipids core), the white areas are calcified plaques. 


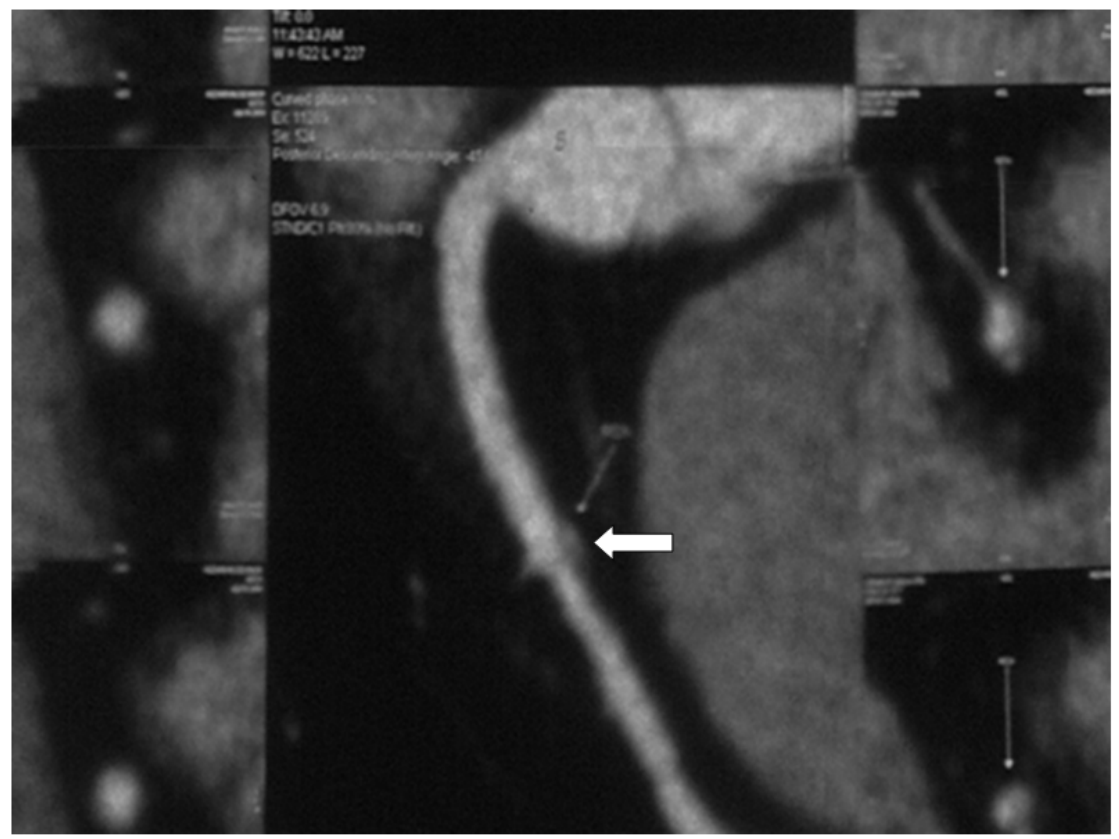

Figure 4. Adventitial soft plaque on the right coronary artery of a 48 years old man (is shown white arrow).

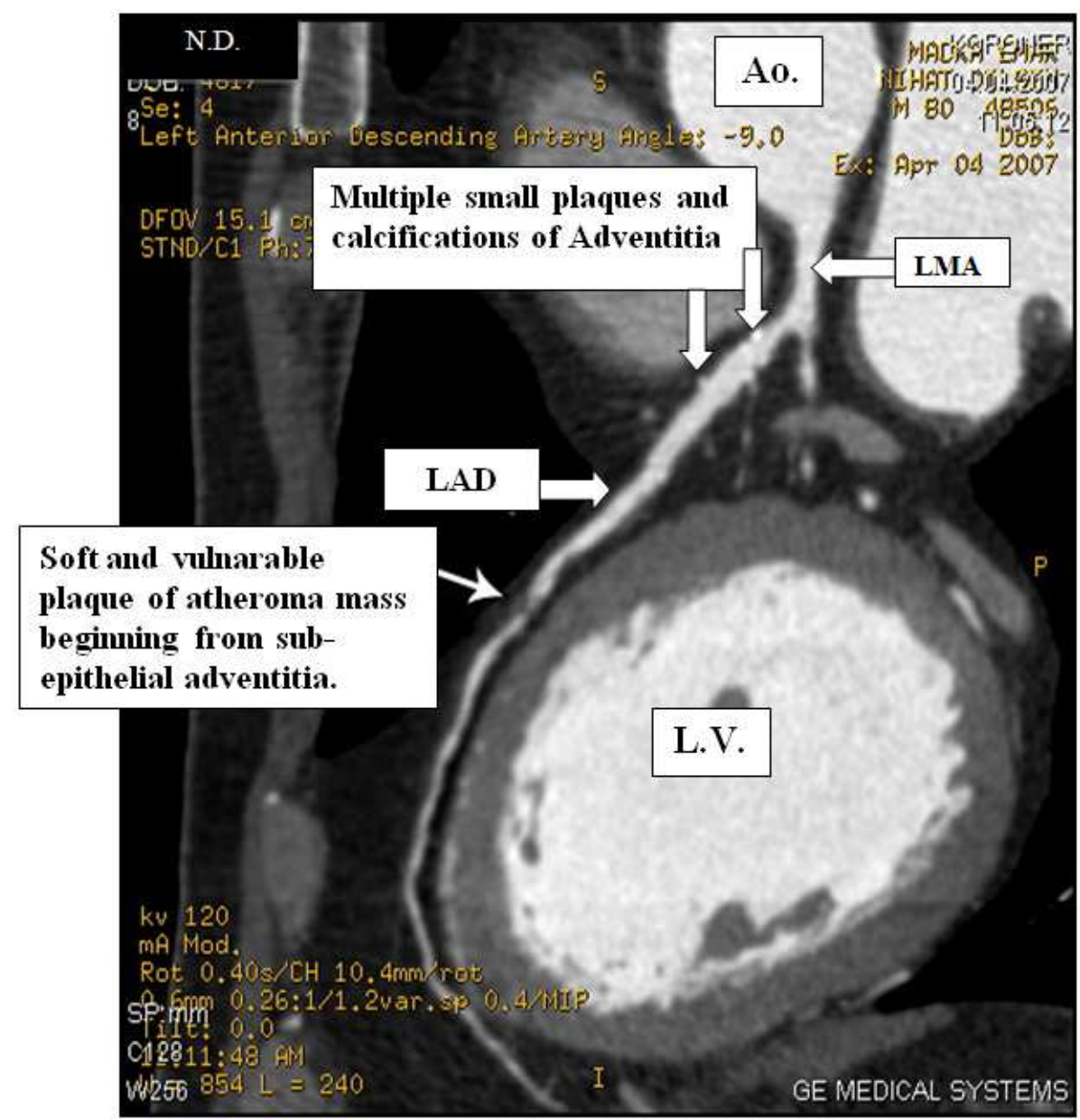

Figure 5. The adventitial atherosclerotic plaques with minimal calcifications on the LAD artery without stenosis and a vulnerable soft plaque of atheromatous component starting from subepithelial area of adventitia on the mid LAD artery with significant stenosis. Ao: Aorta, LAD: Left Anterior Descending, LMA: Left Main Artery, LV: Left Ventricle. 


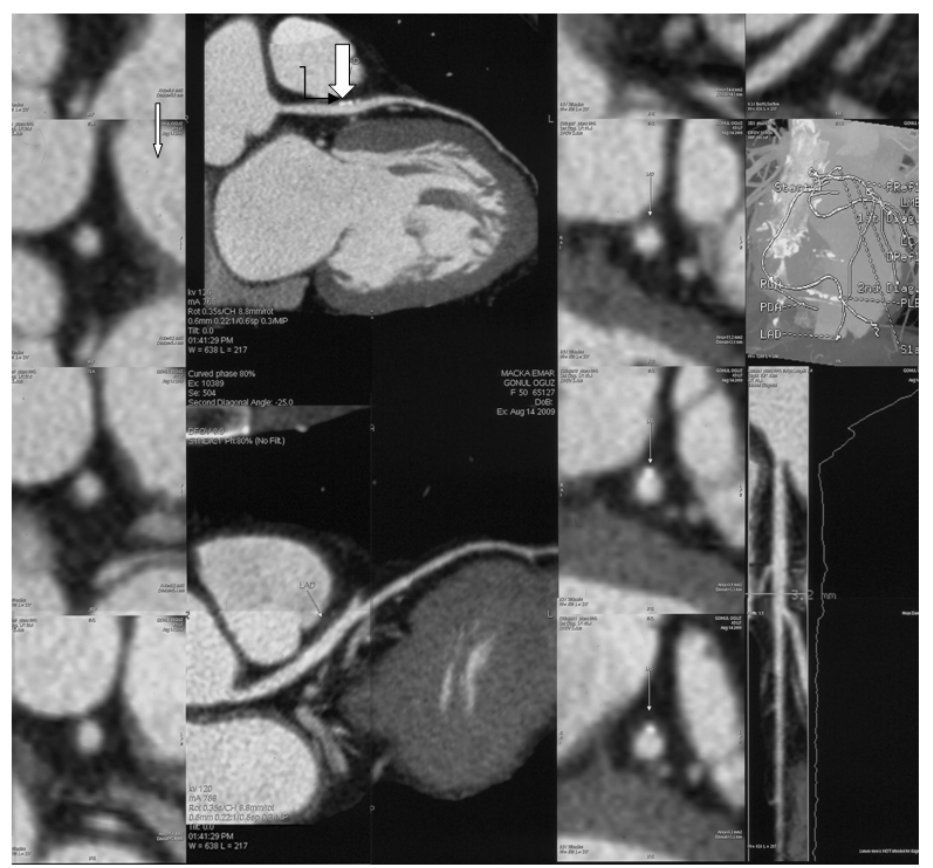

Figure 6. The atheroma mass; minimal calcifications surrounded by some degree of (lipids) soft plaques (have been shown with black and white tiny arrows) were taken place just below the adventitia of LAD artery. (In this case, LAD and Cx arteries were emerging from two separated orifices as a congenital coronary anomaly.)

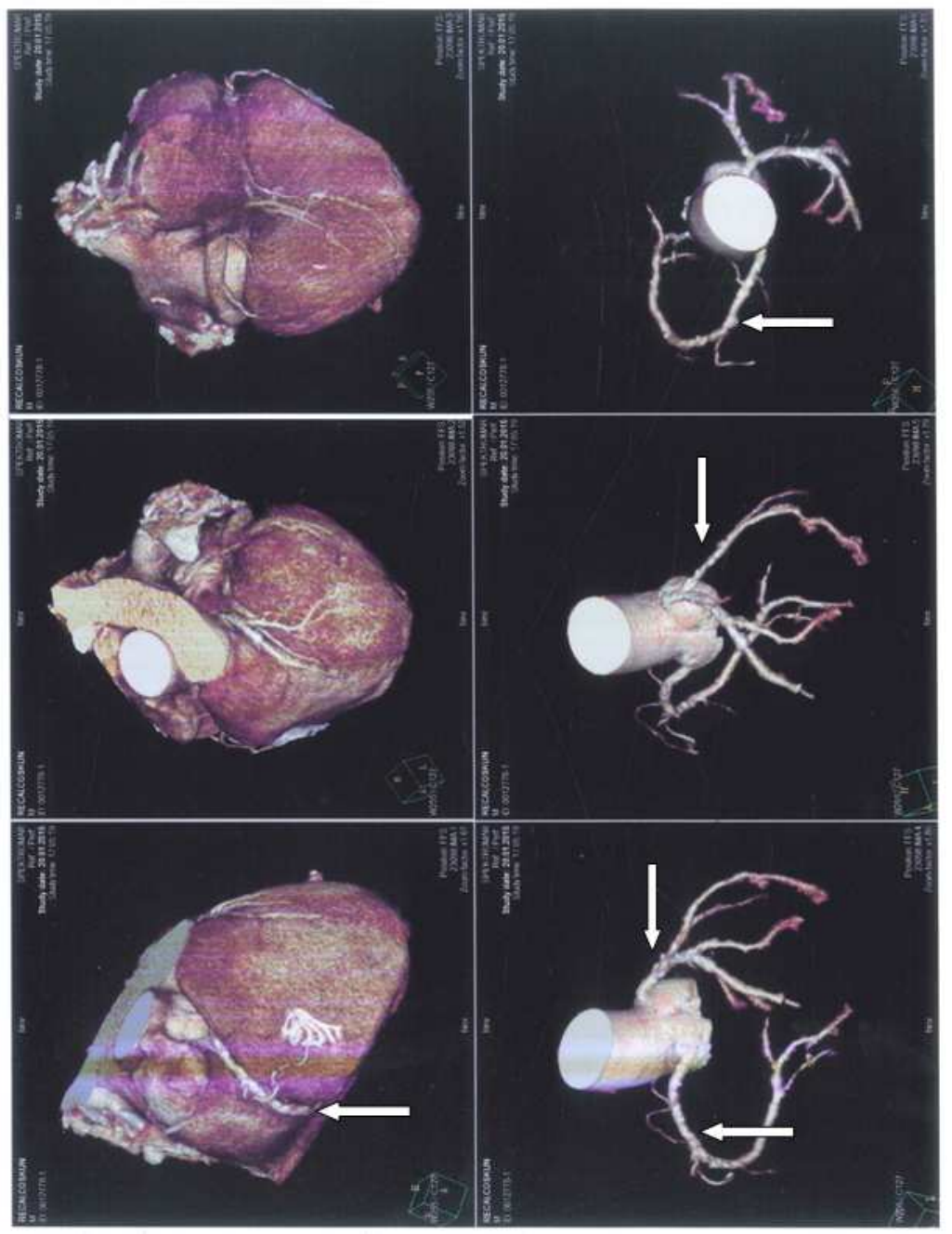

Figure 7. Multi-size adventitial atherosclerocalcifications on the LAD and Cx arteries. 


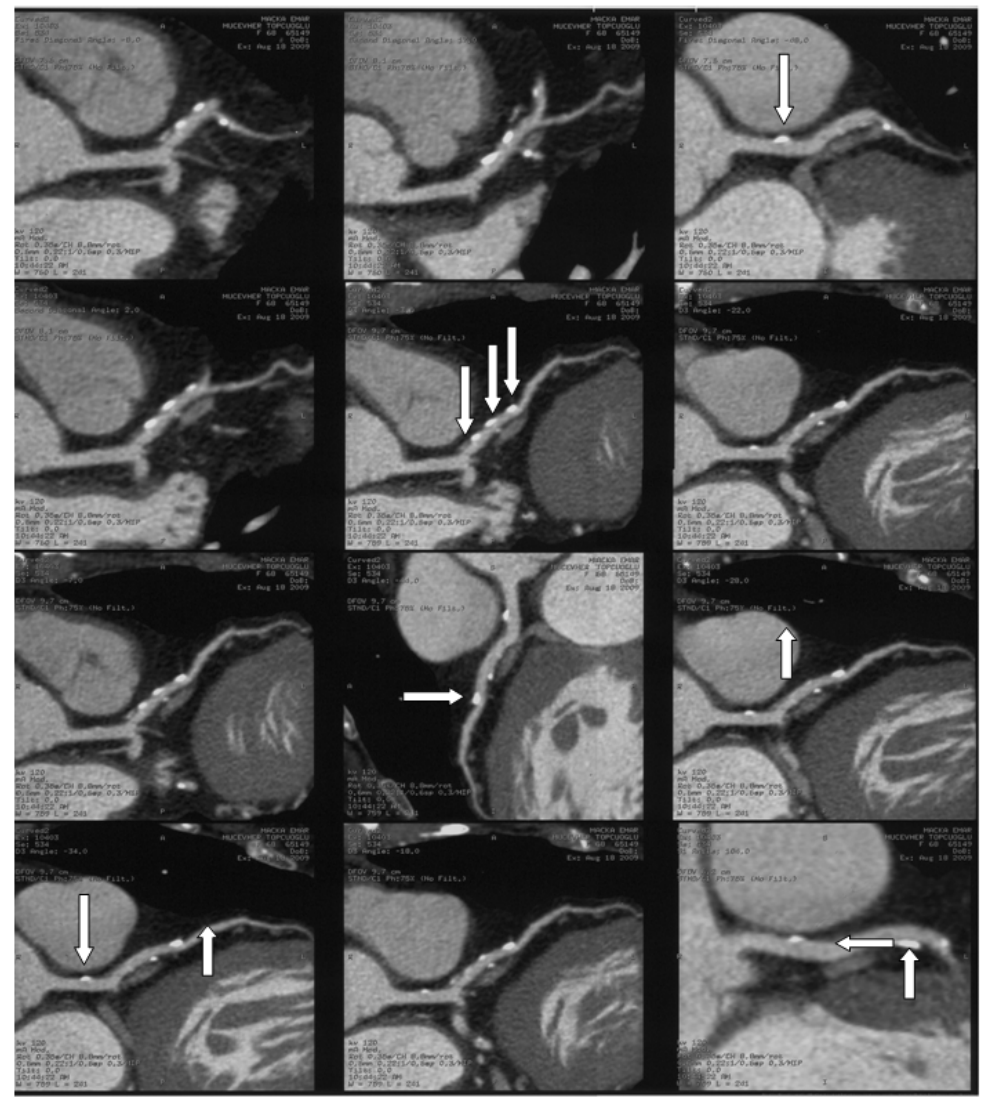

Figure 8. Consecutive many epithelial calcifications looks like beads on the adventitia of the LAD artery and one calcified plaque on the adventitia of Left Main Coronary Artery.

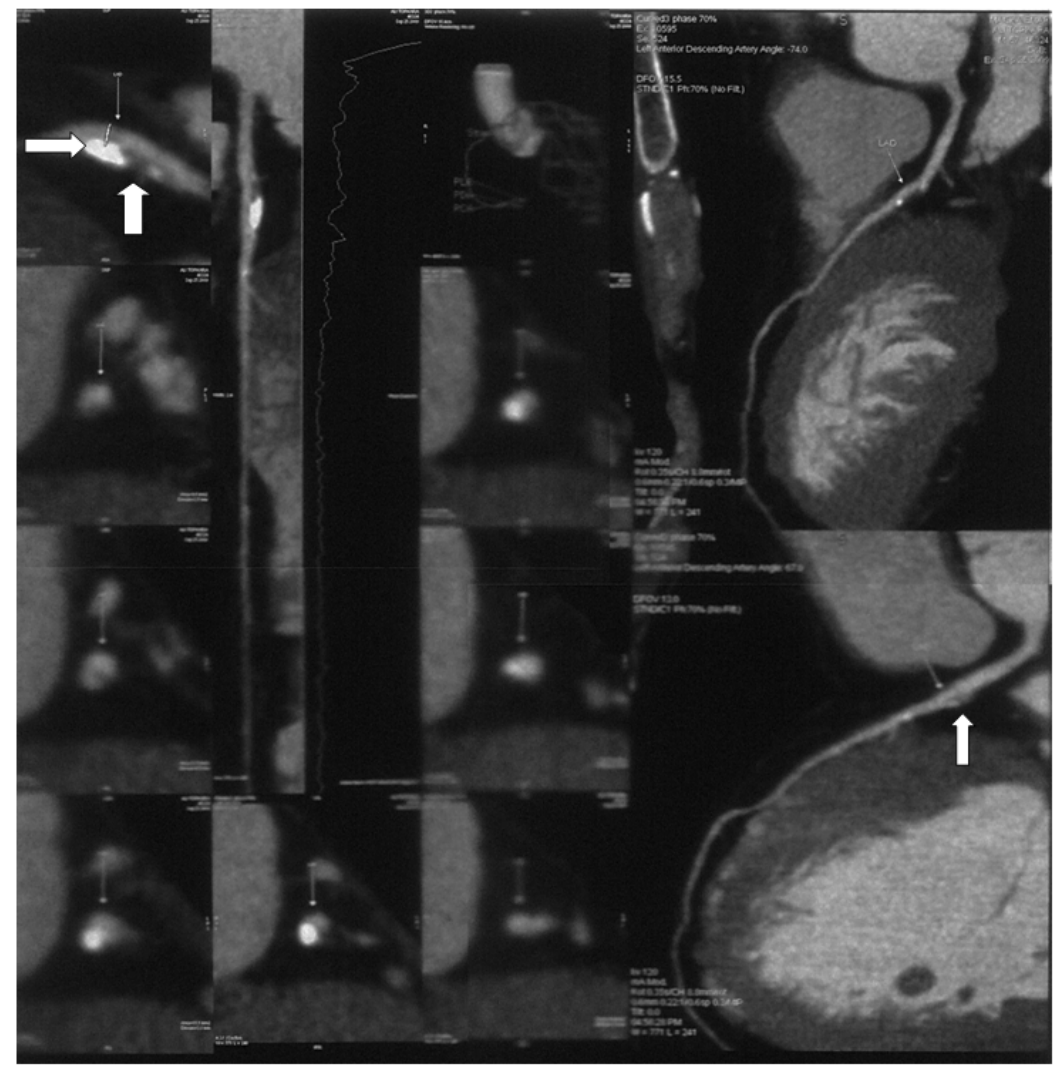

Figure 9. The atheromatous components are shown, with the vertical arrows (lipids core) and medium size adventitial calcifications (the last stop of the atheromatous process) is shown a horizontal arrow on the LAD artery. 


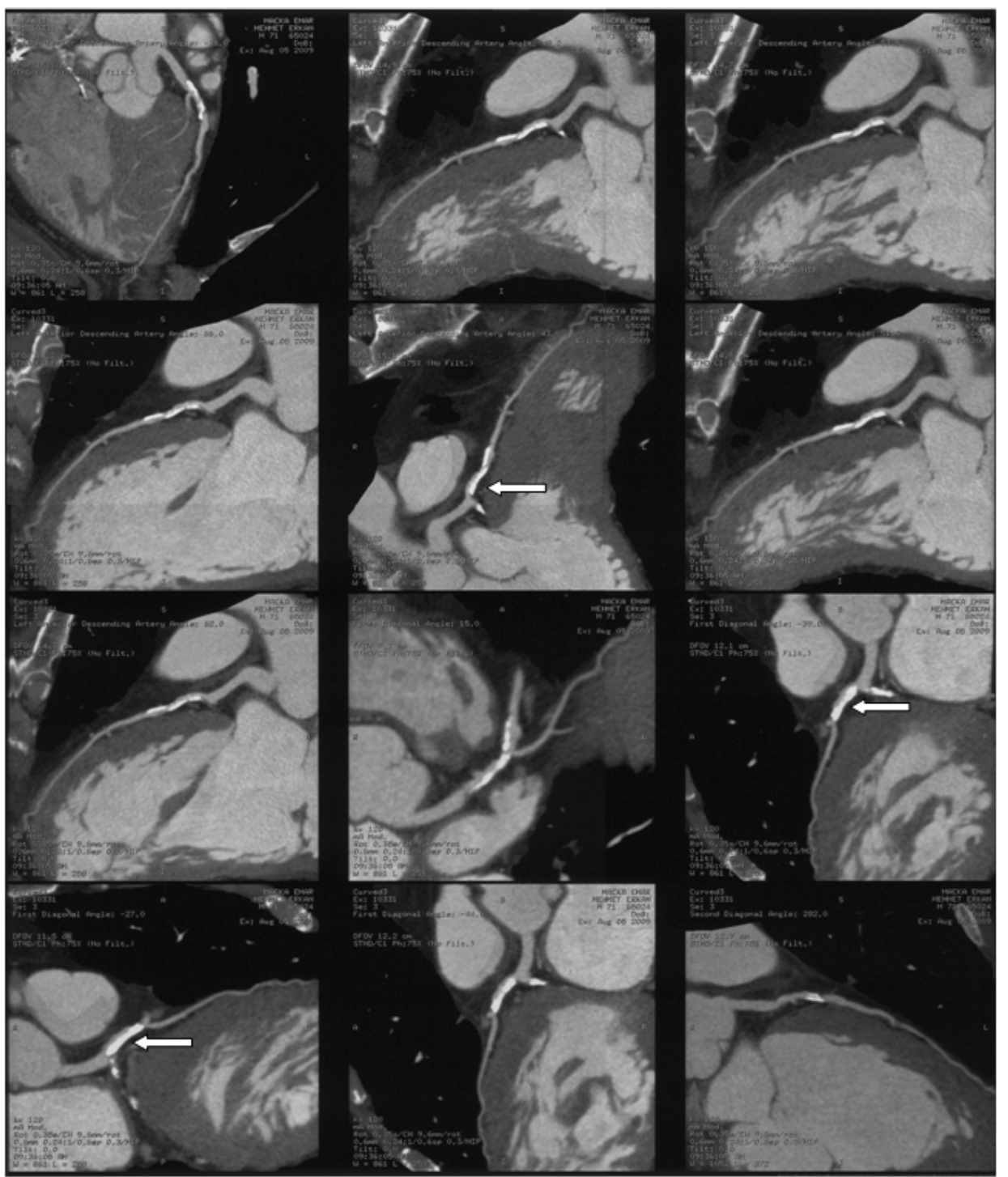

Figure 10. Severe calcification (shown arrows) with significant narrowing of the LAD and Circumflex (Cx) lumens of arteries beginning from adventitia of the vessels.

In our studies, a total of 4055 patients were investigated by MSCT, and invasive coronary angiography were performed to make a comparison between their results. The 3163 patients were male and 892 were female of total patients. The age of the patients were between 33 and 78 years old. At the end of this investigation, 792 patients had stent implantations, 322 patients underwent coronary bypass surgery and 2941 patients were treated medically. The group of 4055 patients underwent MSCT and invasive coronary angiography, and more than 2000 had IVUS coronary arterial biopsy in the operating room, with the cut coronary materials being sent immediately for light, electron and SEM microscopic investigations, which were performed to determine the localization of the atheromas, atheromatous components and calcifications.

Dr. Erzengin gave four international lectures about the formation of atherosclerosis. One of them was at the $78^{\text {th }}$ EAS Congress in Hamburg, Germany, on June 20-23, 2010, while the second of them was at the $3^{\text {rd }}$ World Medicine
Congress in Nalchik KBSU, Russia, on September 20-23, 2010. The others were given in Turkey at the International Congresses of Cardiology in 2011 and 2012[20, 21]. The title was; a new, unknown and a surprise localization of atherosclerosis (Erzengin's Adventitial Atherosclerotic calcification) [21] and Erzengin's Polypill [23]. Atherosclerotic calcification is one of the major causes of progressive degradation of the human arteries and target organs. It is important to note that the primary cause of arterial atherosclerotic calcification has not yet been elucidated. It progresses insidiously, and it damages to the human body remain unknown for along time before symptoms and ischemia associated with the cardiac, cerebral, peripheral and other organs develop. Ischemia creates an imbalance between oxygen supply and demand of the tissue, and is usually related to atherosclerotic calcification obstruction of the arteries, with more or less severe luminal stenosis, that may eventually be complicated by clot formation. Knowledge of its pathogenesis and of the 
early warning symptoms is imperative for interrupting the progression of this disease that not only has high mortality, but is also the cause of various disabling diseases. It has been shown that the cell biology of the arterial wall suggest that injuries of the endothelium, induced by the concurrent action of mechanic and humoral factors, stimulate the formation of atherosclerotic calcifications.

It is currently known that atherogenesis is influenced by more than 250 factors. Atherosclerosis was defined for the first time by Osler in 1908 [9]. Osler mentioned that inflammation and infection were probably responsible for the etiology of atherosclerosis. Following this, the classical endothelial pathway of the atherosclerosis was accepted as the reason of atheroma formation in 1995 by AHA. Until today, it has been accepted that atherosclerotic calcification is located in the subendothelial area (intimae). Only Mönckeberg demonstrated several years ago the localization of atherosclerosis in the media among a limited group of patients $[9,23]$. According to the classical approach; it was known that this progressive process starts and grows up from intimae, which is inside the lumen of arteries, and also mostly progresses as time passes.

Ethics approval of this study was given by the ethics committee of the University of Istanbul in 1998. Also, any of this manuscript have no conflict of interest.

\section{Results and Discussion}

However, our team recently demonstrated the adventitial localization of atherosclerotic calcifications by using the 64-320-640 Multi Slice Computed Tomography (MSCT) with a magnifying glass on 4055 patients. Our conclusion was that the MSCT 320 is a very useful technique for the characterization of human coronary plaques morphology, for reason that the MSCT can non-invasively determine tissue density within lesions. In addition, as a unique and non-invasive method, the MSCT is used for the detection of coronary atherosclerotic plaque morphology (vulnerable plaques, in particular) and calcification scores, also for the diagnosis of silent ischemia and asymptomatic myocardial infarctions caused by lumen narrowing due to calcification starting from the adventitia[19].

Thus, our surprising findings were that the formation of the atherosclerotic and calcified plaques begin and develop not only in the intimae or media (Mönckeberg's Sclerosis), but also on the adventitia (Erzengin's Arterial Atherosclerotic Calcifications) of the coronary arteries and/or all of the medium and large arteries [21]. We suggest that the formation of the Adventitial Atherosclerotic calcifications is caused by vasa-vasorums and/or diffusion from the adipose tissues around the pericardium. White and brown adipose tissues around the heart contain more than 250 atherogenic substrates such as macrophages, oxLDL, etc $[1,2,3,4]$.

As a result of our studies, these views were generated step-by-step as our knowledge has increased and become more refined during the last 5 years. It was the first time in the literature that the Adventitial Atherosclerotic Calcification was demonstrated by MSCT and proved pathologically in our research laboratories. In addition to this, coronary angiography, IVUS, perioperative findings, histopathological data detected by light, electron microscopy and environmental SEM have been used for verifying the results.

Regardless of the way the atheromatous component formation occurs, or which tunicas of the arteries they develop, the result is still almost always same. Formation of the atheroma mass depends on genetics, hypercholesterolemia, diabetes, smoking, hypertension, abdominal obesity, sex, aging, hyperuricemia, chemical irritants, infections, high svCRP and fibrinogen levels, circulating amines, and immune complexes [7].

Atherosclerotic calcification is one of the major causes of progressive degradation of the human arteries and target organs. It progresses insidiously and its damaging effects of human organism remain unknown for a long time before cardiac, cerebral or peripheral symptoms and/or other ischemia develop.

For many years, atherosclerotic plaque formation has been accepted as a dynamic, progressive process and a very dangerous fatal disease that can never be effectively prevented, stabilized or treated because of its dangerous progression. Unfortunately, atherosclerosis is still associated with high morbidity and mortality rates across the world.

According to our new clinical and laboratory findings; we believe that atherosclerotic and calcified plaques can be stabilized and fully treated with only drug therapy, regardless of where their localization is on the arteries. This means that, in addition to the current treatment, our polypill combination can totally cure the atherosclerosis and prevent plaque formation permanently. So, we have proved that this insidious and potentially fatal process is regressive, preventable and totally curable using only a combined drug therapy.

In this context, we will write about a new additional treatment (Erzengin's Polypill) for atherosclerotic calcifications [23, 26]. Here, we will present Erzengin's new medical combination therapy against this progressive, dynamic and potentially fatal disease. While Table I shows this Erzengin's Polypill combination therapy, Table II depicts mechanism of this Erzengin's Polypill combination therapy. Atherosclerotic calcifications lead to death and disability by occluding the arteries of vital organs. The noninvasive treatment prevents this severe diseases, which otherwise requires expensive, often palliative and high-risk surgical procedures and/or invasive interventions. The medical treatment would also be necessary after CABG surgery or stent implantation in order to prevent the progression of the virulent process. Erzengin's combination therapy also makes a major contribution to the treatment of osteoporosis while it prevents diseases such as atherosclerotic calcified arteries, calcified cardiac valve diseases, calcified constrictive pericarditis and urinary 
phosphate calculus. We found that the side effect of this drug was headache. Fortunately, it was unimportant because we observed less than $2.3 \%$ patients. Any bleeding and obstruction was not seen any of the patients. The main purpose of our therapy with Erzengin's Polypill is to prevent the formation of atherosclerosis, eliminate the existing atherosclerosis, and to completely heal the existing disease.

Table I. Erzengin'sPolypill combination.

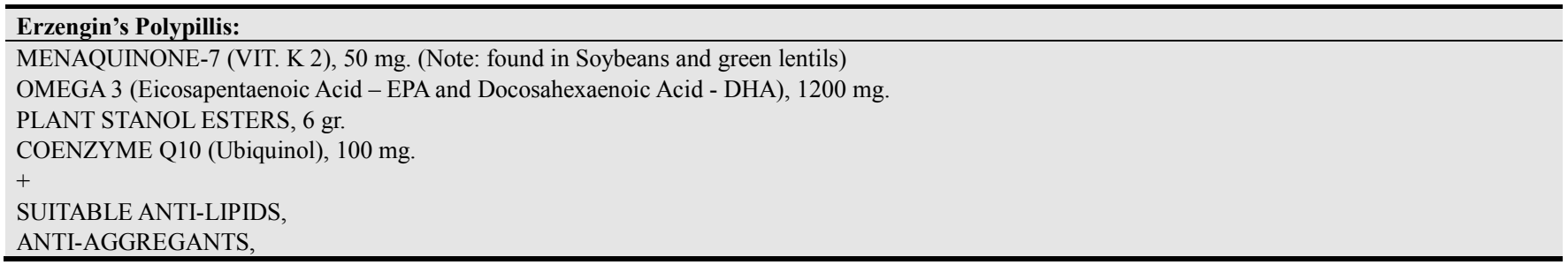

In the light of recent developments and new knowledge, suitable anti-lipid and anti-thrombotic treatments are approved for suitable patients. In addition to these treatments, it is known that Menaquinone-7 (Vitamin K2, found in Soybeans and green lentils) [9] and Vitamin D3 causes the arterial calcifications regress, and the calcium to mobilize from the walls of arteries to the bones. As it is well known, Vitamin $\mathrm{K}$ includes 2 different groups of agents; Phylloquinone (Vitamin K1) and Menaquinone (Vitamin K2). Insufficient ingestion of Vitamin K2 causes atherosclerotic and calcified plaques on the arterial walls and atherosclerotic coronary arteries due to the lack of Matrix Gla-Protein (MGP) carboxylation. This means that sufficient ingestion of Menaquinone-7 (especially Vitamin K2 = MK-7) prevents atherosclerotic and calcified plaque formation. The results of
Menaquinone-7 treatment are controlled by using MSCT, besides Clopidogrel $75 \mathrm{mg}$ /daily should also be added to this treatment routinely. In addition, according to several studies, it has been observed that the ingestion of Plant Sterol Esters and particularly the esters of Plant Stanol lowers total cholesterol, LDL and triglyceride in serum and cures atherosclerosis in the ratio of $15-27 \%$. The combination of Plant Stanol Esters and statins prevent atherosclerosis in the ratio of $46 \%$.According to our results; Plant Stanol esters are more effective than sterols. On the other hand, these esters prevent side effects of statins. The other two components of Erzengin's polypill combination are Omega-3 (Eicosapentaenoic Acid - EPA and Docosahexaenoic Acid DHA) and Ubiquinol (Coenzyme QH).

Table II. The mechanism of Erzengin's Polypill.

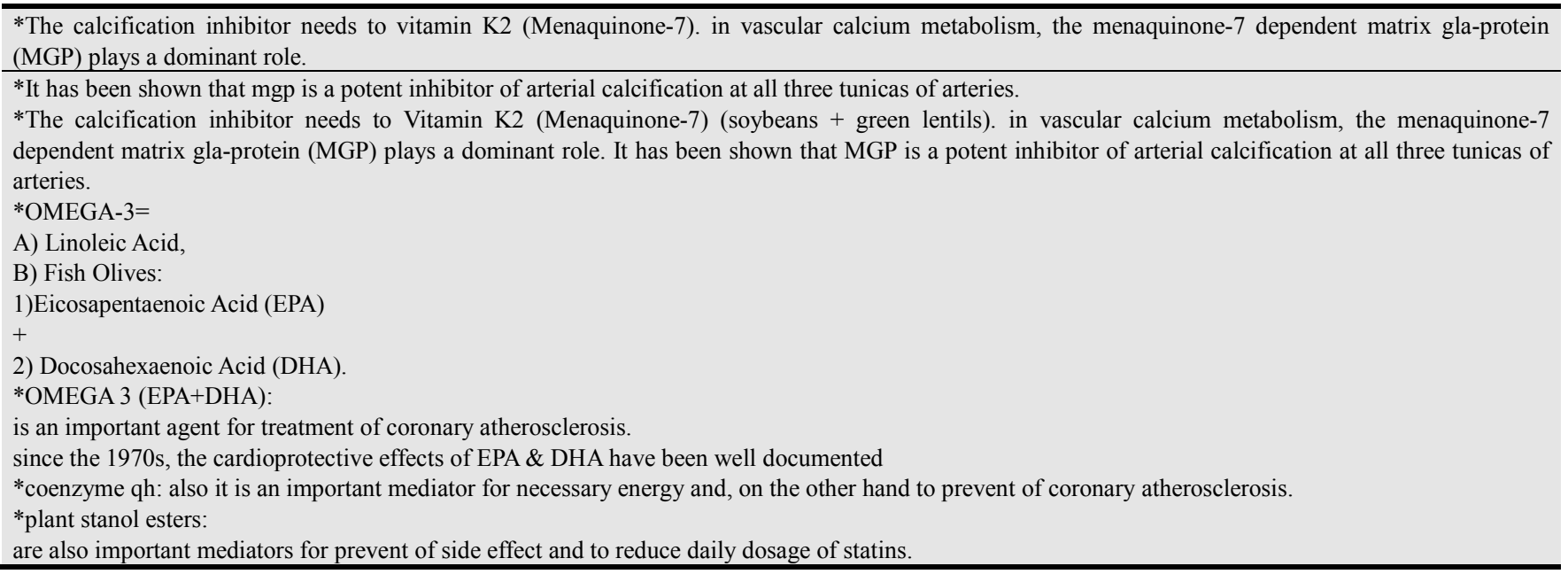

At the beginning of our studies, the comparative animal study (Stage A) was initiated at DETAE in Istanbul Medical Faculty (Ethics approval of this study was given by the ethics committee of the University of Istanbul).

For the second stage of our studies, we planned a case study group (Stage B) consisting of patients with informed consent and divided into 2 random subgroups. The first subgroup (Group B-1) consisted of patients who preferred to receive ordinary treatment. These patients were treated with conventional medication in order to allow comparison with the second group of patients. This subgroup (Group B-2) were medicated with the polypill combination therapy, together with the other ordinary therapy.

Furthermore, in this new study, there are more patients than the previous study andall patients underwent stage B procedure.

If the studies accomplish their objectives, there will be a permanent solution for the atherosclerosis that leads to severe diseases in arteries of vital organs and sudden deaths. The combination of Erzengin's Polypill together with the ordinary 
therapy will sort out the complications of the atherosclerosis permanently. We believe that this therapy will completely prevent the atherosclerosis in a non-invasive, risk-free and inexpensive way. Our research will make an incomparable contribution to all of humanity by expanding the life span and enhancing quality of life. It will represent a remarkable success in the name of science, and make a great contribution to the economy.

We would like to introduce a special four cases diagnosed by MSCT which two of them silent ischemia, one of them acute MI, one of them angina pectoris with myocardial bridge (respectively, a 88 year old man, 62 year old man, 66 year old man and 53 year old woman) in total 4055 patients. First three patients also underwent for diagnosing directly MSCT because of being afraid of invasive angio.

First patient was admitted to the Cardiology Department with trivial exercise dyspnea, and palpitation from time to time lasting for about one month in 2007. Electrocardiogram (ECG) and Exercise ECG were normal (Figure 11). The patient denied coronary angiography, and 640-slice MSCT was performed. With coronary angiography, we identified a left anterior descending coronary artery (LAD) with vulnerable soft plaque leading to severe coronary artery stenosis $(95 \%)$ at the middle segment; this observation was in parallel with the MSCT findings. Percutaneous coronary intervention (PCI) was performed for LAD lesion, and a drug-eluting stent was implanted after predilatation. The patient was examined routinely and is completely asymptomatic for more than six years.

Second diabetic patient was admitted with trivial swelling after routine insulin injection. His cardiac enzymes were elevated which were taken routinely before admittance to our clinic. His ECG (Figure 12) showed slightly prominent and non-significant T waves on V2-3 leads. On MSCT (320 slice), we detected total occlusion of the left circumflex artery proximal segment and critical stenosis (98\%) at the middle segment of LAD. Invasive coronary angiography was performed on the same day. The coronary angiography results was in agreement with the MSCT findings. This patient has had an acute asymptomatic posterior myocardial infarction. PCI with predilatation was performed firstly for the completely occluded circumflex artery, which was opened successfully. A drug-eluting stent was then implanted. Again, predilatation was performed to the critical LAD lesion and drug-eluting stent was implanted. The patient is followed up routinely. He is asymptomatic for more than three years after the procedure.

The third patient, a 66-year-old man, was examined routinely without any complaints. His resting ECG was normal. However, during the $4^{\text {th }}$ stage of the ECG exercise, an atypical chest pain occurred without ST-T changes. His MSCT (320 slice) surprisingly showed 95\% narrowing LAD lesion with adventitial calcifications (Figure 13). This lesion was similar to his coronary angiography. A metal bare stent implantation was performed. Since then, he has been very active without any complaints or symptoms.
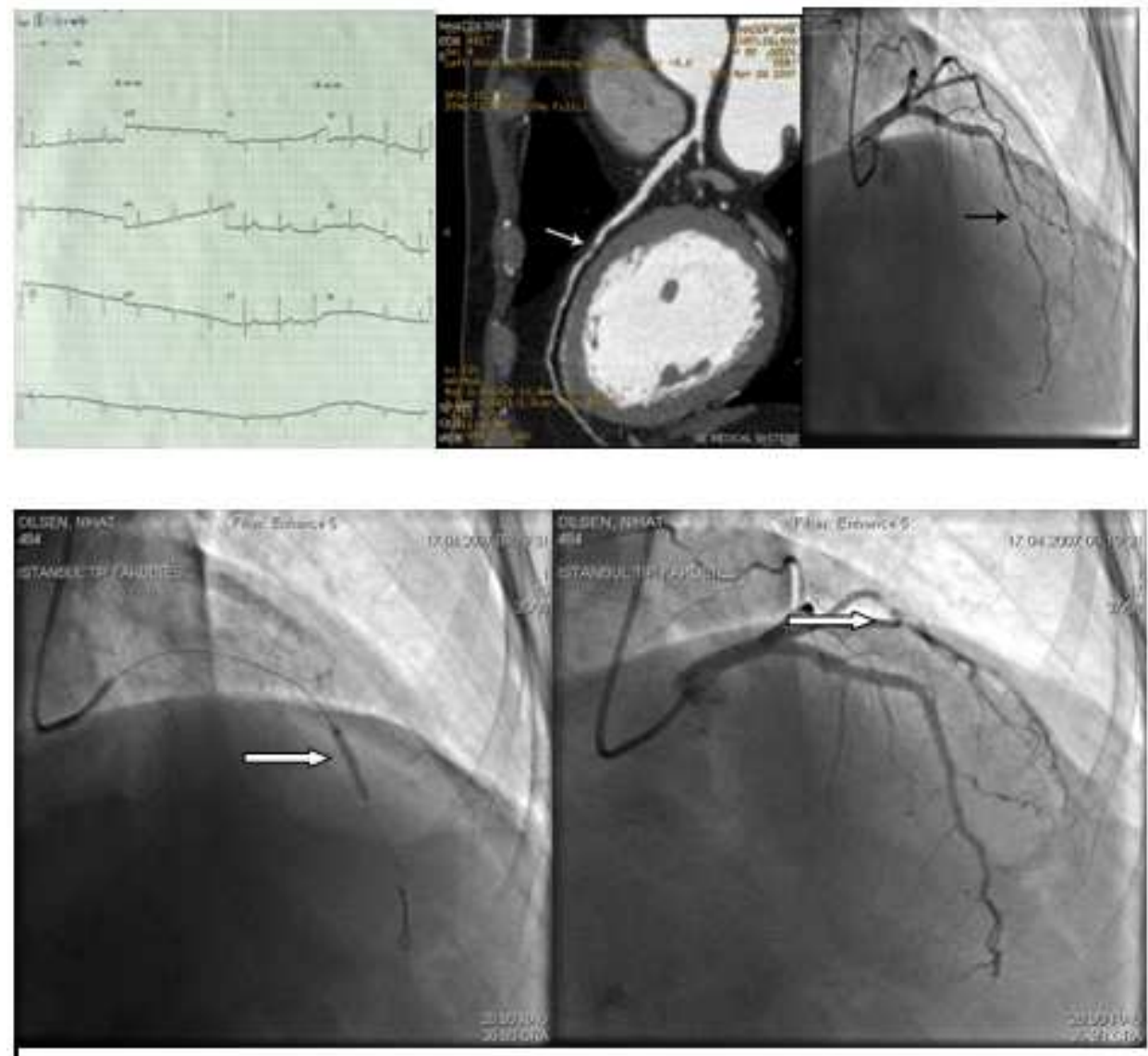

Figure 11. This figure shows first patient's ECG, MSCT, C. Angiography and stent implantation. 

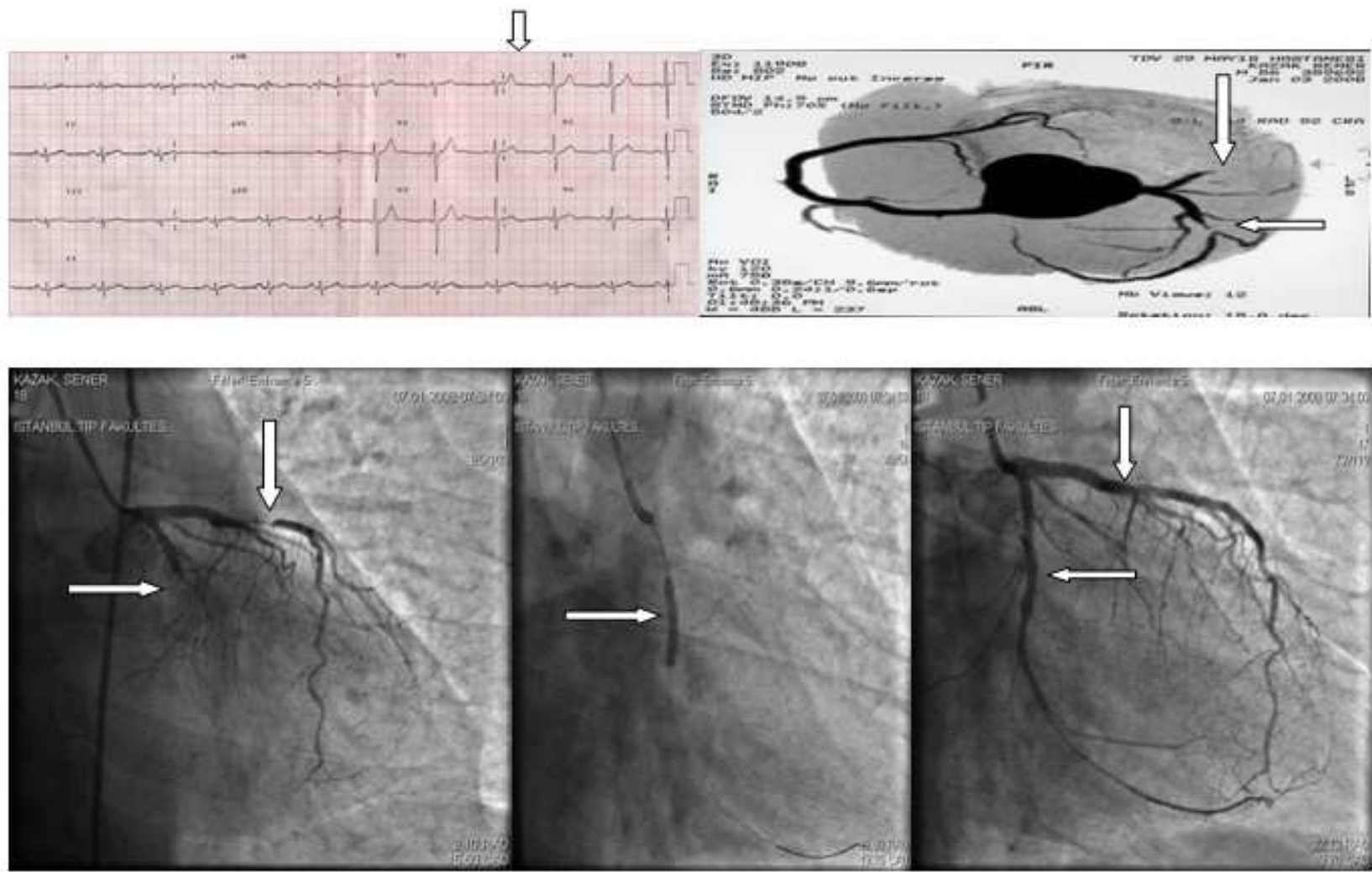

Figure 12. This figure illustrate ECG, MCCT, C. Angios and stents implantation of totaly ocluted Cx and strictly stenosed LAD lesion of case 2.
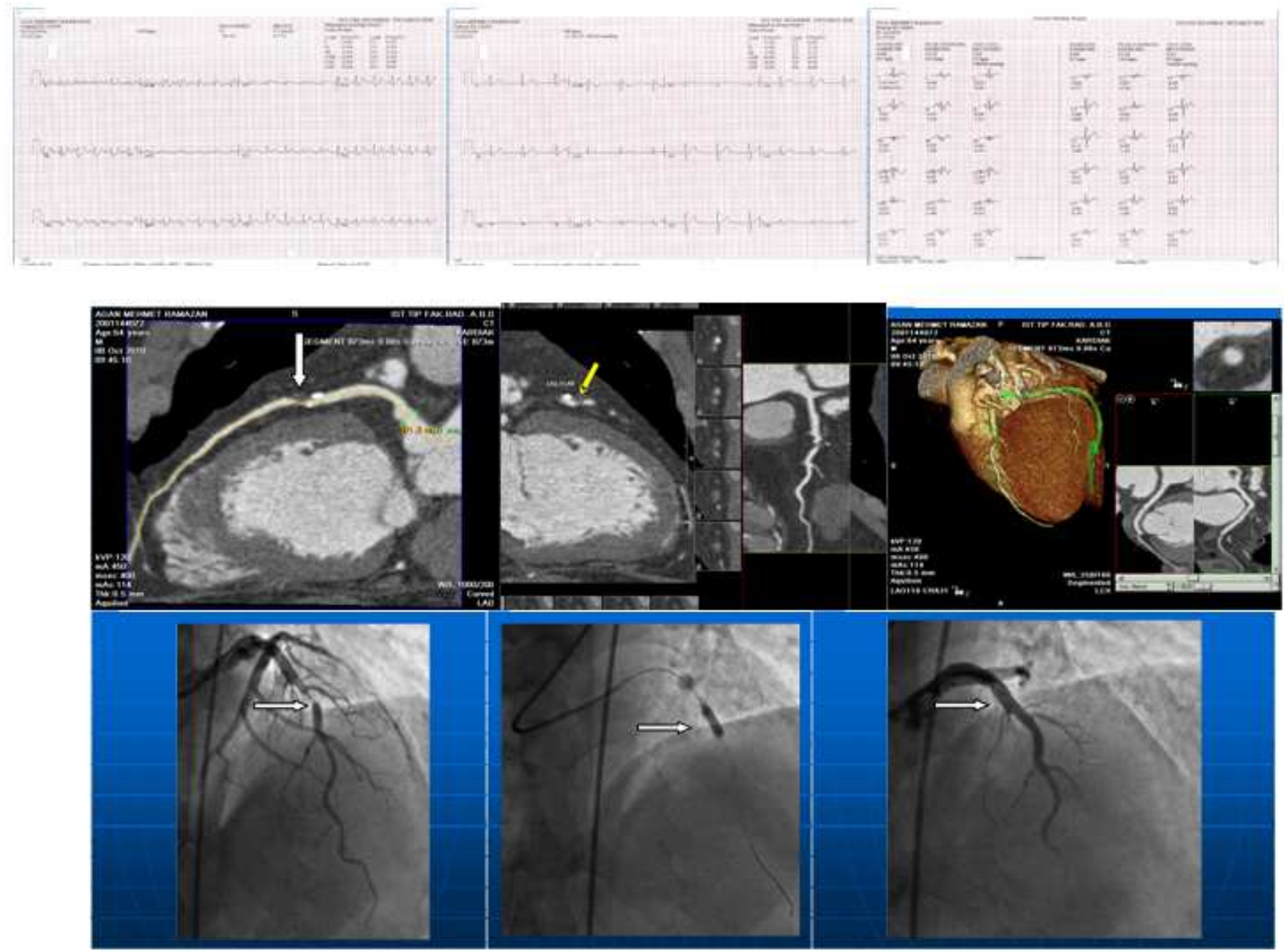

Figure 13. This figure shows ECG, Exercise ECG, MSCT, C. Angios and after stent of LAD artery of case 3. 


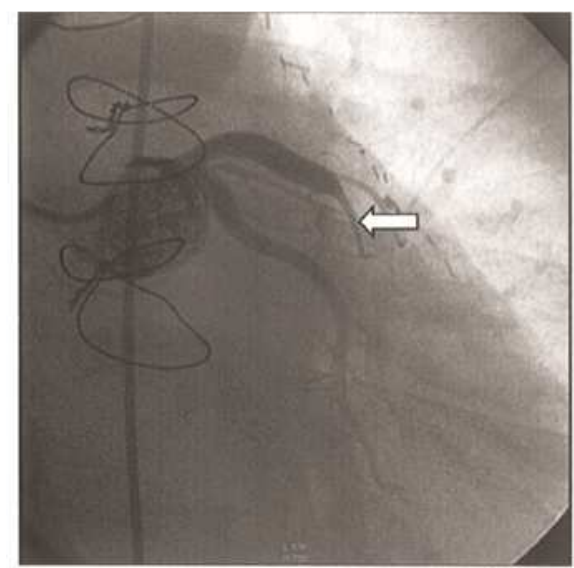

a)

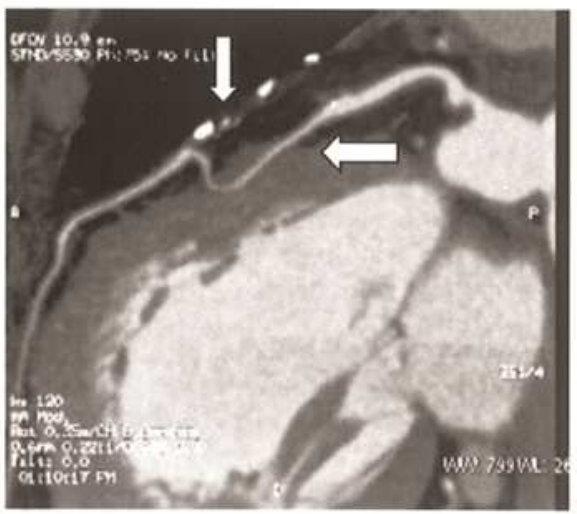

c)

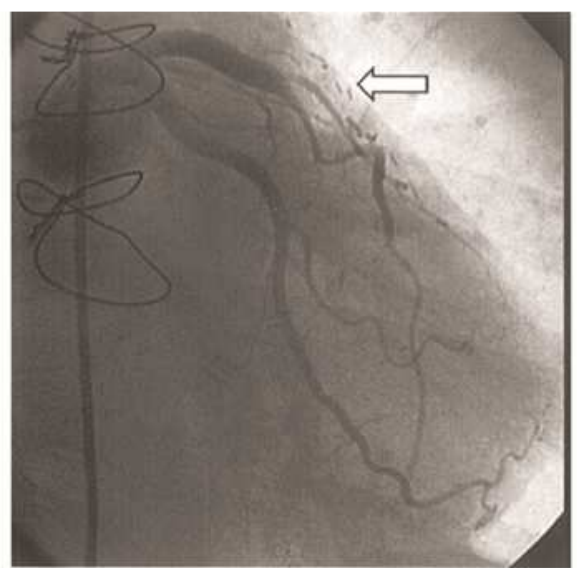

b)

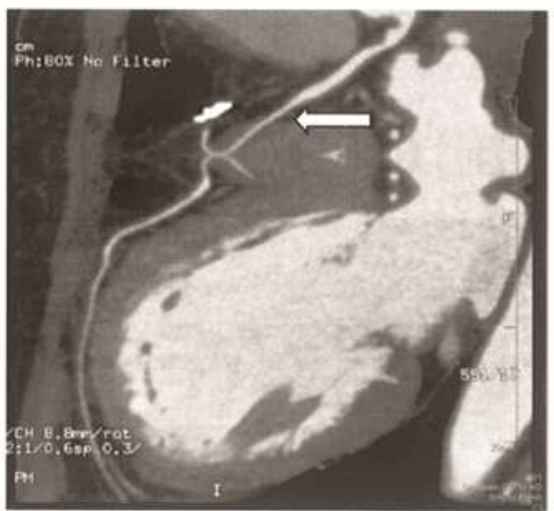

d)

Figure 14. This figure shows that case 4; occluded LAD artery and suspected myocardial bridge (a, b) in the conventional angios. The Hook Shape (Erzengin's Cardiac CT Sign) of the LAD in the myocardium (c) and occluded LIMA due to competition (myocardial systolic high pressure of LAD) (c, d) was accurately diagnosed myocardial bridge with MSCT.

The fourth patient, a 53 year old lady was operated CABG operation to the LAD artery implanted LIMA for coronary stenosis by mistake in a private hospital 9 years ago. This patient suffers from angina pectoris after operation until today. We admitted the patient recently and performed conventional coronary angios. We diagnosed that LIMA artery occluded and suspected myocardial bridge of the LAD. This patient underwent Cardiac CT (MSCT 320 slice) for recognition of myocardial bridge and diagnosed typical "Hook shape (Erzengin's CT Sign)" of the LAD artery (Figure 14). This finding is gold standart for myocardial bridge [9]. During systolic myocardial contraction, increased pressure of the LAD occluded LIMA bypass greft (with competition). Patient treated with beta blockers and stopped nitrogliserines which were prescribed previously out of our clinic. Patient followed up in our outpatient clinic last 8 months without any complaints and she has been doing well.

\section{Conclusion}

We conclude that the MSCT is a very important tool for the diagnosis and evaluation of coronary lesions. In addition, it is an important guide for curing silent ischemia and myocardial infarction, particularly for patients unwilling to undergo invasive coronary angiography.

Today;

It is possible to treat coronary (or all arteries). Atherosclerotic calcifications can be treated medically with only drug combination therapies.

(An innovative treatment and exact solution for coronary atherosclerotic calcifications)

\section{References}

[1] Fuster V. Epidemic of cardiovascular disease and stroke: The three main challenges. Circulation 1999; 99:1132-1137.

[2] Fuster V. Atherosclerosis, thrombosis, and vascular biology. Cecil Textbook of Medicine, Ed: Lee Goldman, Dennis Ausiello, 22 ${ }^{\text {nd }}$ Edition, Saunders, Philadelphia, Philadelphia, Pages 383-400, 2004.

[3] Lipid Study Group. Prevention of cardiovascular events and death with pravastatin in patient with coronary heart disease and abroad range of initial cholesterol levels. The Long-Term Intervention with Pravastatin in Ischaemic Disease (LIPID) Stud Group. N Eng J Me 1998; 339:1349. 
[4] Falk E, FusterV. Atherogenesis and its determinants. Hurst's The Heart, Ed: Valentin Fuster, R. Wayne Alexander, Robert A O'Rourke, Robert Roberts, Spencer B. King III, Hein J.J.Wellens. 10th edition, McGraw-H1ll, New York, Volume 1 Pages 1065-1093, 2001.

[5] Ross R. The biology of atherosclerosis. In:Topol E J, ed. Comprehensive Cardiovascular Medicine. Philadelphia:lippincott-Raven, 1998:13.

[6] Iiyama K, Hajra L, Iiyama M, Li H.Patterns of vascular cell adhesion molecule-1 and intracellular adhesion molecule-1 expression in rabbit and Mouse atherosclerotic lesions and at site predisposed to lesion formation. Circ Res 1999; 85,:199207.

[7] Expert Dyslipidemia Panel of the International Atherosclerosis Society Panel members. An International Atherosclerosis Society Position Paper: global recommendations for the management of dyslipidemia - full report. J Clin Lipidol, JanFeb 2014;8(1), 29-60.

[8] Lesckha S, Alkadhi H, Plass A, Desbiolles L Grunenfelder J, Merincek B, Wildermuth S. Accuracy of MSCT coronary angiography with 64-slice technology: Firs experience. Eur Heart J 2005; 1482-1487.

[9] Erzengin F, Büyüköztürk K. Cardiac Imaging in Textbook of Internal Medicine, Ed: Kemalettin Büyüköztürk, Faruk Erzengin et all. Nobel, 2007, Istanbul, 1623-1644.

[10] Westendorp RG, Assendelft WJ, Elzen WP, Cessie S, Gussekloo J; Use of Framingham risc and new biomarkers to predict cardiovascular mortality in older people: Population base observational cohort study. BMJ. 2009, 8; 338: a 3083.

[11] Third Report of the National Cholesterol Education Program (NCEP) Expert Panel on Detection, Evaluation and Treatment of High Blood Cholesterol in Adult (Adult Treatment Panel III) final report. Circulation, 2002; 106:3143-421.

[12] Conroy RM, Pyorala K, Fitzgerald AP, Sans S, Menoti A, De Backer G: Estimation of ten year risk of fatal cardiovascular disease in Europe: The SCORE Project. European Heart Journal, 2003, 290: 898-904.

[13] Hendel RC, Patel MR, Kramer CM, Poon M. ACC/ACR/SCMR/ASNC /NASCI/ SCAI/SIR Apropriateness Criteria for Cardiac Computed Tomography and Cardiac Magnetic Resonance Imaging. Journal of the American College of Cardiology, 48 (No.7); 1-23, 2006.

[14] Achenbach S, Daniel WG: Computed Tomography of the Heart. Braunwald's Heart Disease, 7th edition, Elsevier Saunders, Pensilvania,USA, 2005, 285-287.

[15] Higgins CB, de Roos A: MRI and CT of the Cardiovascular system. Lipincor \&Williams and Wilkins,USA and the Netherlands, 2006.
[16] Achenbach S, Moselewski F, Ropers D, Ferencik M, Hoffmann U, MacNeill B, Pohle K, Baum U, Anders K, Jang IK, Daniel WG, Brady TJ. Detection of calcified and noncalcified coronary atherosclerotic plaque by contrastenhanced, submillimeter multidetector spiral computed tomography: a segment-based comparison with intravscular ultrasound. Circulation 2004; 109:14-17.

[17] Sangiorgy G, Rumberg J A,Severson A, et al. Arterial calcificationand not lumen stenosis is highly correlated with atherosclerotic plaqueburden in humans: A histologic study of 723 coronary artery segments using nondecalcifying methodology. J Am Coll Cardiol 1998; 31: 126-133.

[18] Mintz GS, Pichard AD, Pompa JJ, et al. Determinants and correlates of target lesions calcium in coronary artery disease: A clinicali angiographic and intravascular ultasoun study. J Am Coll Cardiol 1997; 29: 268-274.

[19] Yönal İ, Banzagch M, Özcan M, Adalet K, Erzengin F: Cardiac Computed Tomography; The Unique Noninvasive Method For The Detection Of Coronary Atherosclerotic Plaque Morphology And For The Diagnosis Of Silent Ischemia, Lumen Narrowing Calcification Of Adventitia: With Two Case Reports. The Heart Surgery Forum. A Cardiothoracic Multimedia Journal, Vol.12 (Supl.1), 2009, p:69-70.

[20] Erzengin F:Adventitial Calcification: Surprise Location. Atherosclerotic and calcified plaques begin and grow up not only beneath the endothelium, but also on the adventitia. 78th European Atherosclerosis Society Congress (78th EAS Congress), June 20-23, 2010, Hamburg, Germany.

[21] Erzengin F: Adventitial (Erzengin's) Atherosclrerocalcification: Surprise Location. Atherosclerotic and calcified plaques begin and grow up not only beneath the endothelium, but also on the adventitia. The 3th World Medicine Congress İn abstract book, Page 4 September 20-23, 2010 / NalchikRussia.

[22] Bissen E.A. Plaque Adventitia: Service Hatch or Battleground? 79th EAS Congress Gothenburg, Sweden, June 26-29, 2011.

[23] Erzengin F: Erzengin's Polypill: An additional and new medical treatment of atherosclerocalcifications. International Journal of Cardiology. Volume147, Page 111-112, 2012.

[24] Efe D and Aygun F. Assessment of the Relationship Between Non-alcoholic Fatty Liver Disease and CAD using MSCT. Arq Bras Cardiol, 2014, 102 (1), 10-18.

[25] Lee S, Chang H, Sung J, Kim K, Shin S, Cho I, Shim C, Hong G, Chung N. The Impact of Obesity on Subclinical Coronary Atherosclerosis According to the Risk of Cardiovascular Disease. Obesity, july 2014, 22 (7), 1762-1768.

[26] LIacobellis G, Ribaudo MC, Zappaterrano A,Iannucci CV, Leonetti F: Relation between epicardial adipose tissue and left ventricular mass. Am.J Cardiol, 2004, 94,1084-7. 